\title{
Physiological and structural adaptability of Anemone shikokiana leaves to heterogeneous habitats
}

\author{
Yujuan Pang ${ }^{1}$, Yan Gao ${ }^{1}$, Lei Feng ${ }^{1}$, Bin Jiang ${ }^{1}$, Zhi Wang ${ }^{1}$, and Fuhua Bian ${ }^{1}$ \\ ${ }^{1}$ Affiliation not available
}

May 5, 2020

\begin{abstract}
Anemone shikokiana is distributed in two heterogeneous habitats, including mountaintop shrubland and conifer and broad-leaf mixed forest. To better understand the mechanisms used by A. shikokiana in adapting to these different environments, the photosynthetic efficiency, chlorophyll fluorescence and microstructure and ultrastructure of the leaves were investigated. The findings revealed that, under the same optical quantum flux density, the net photosynthetic rate in the leaves of mountaintop shrubs was significantly higher than that found in the mixed forest, but the stomatal conductance, intercellular CO2 concentrations and transpiration rates were lower. The effective quantum yield of photosystem II (PSII), photochemical quenching coefficient, non-photochemical quenching coefficient and electron transfer rate in the conifer and broad-leaf mixed forest were significantly lower than those values determined for the shrubland plants. However, the maximal quantum yield of PSII exhibited no significant difference between the two habitats. Although leaf thickness was greater and mesophyll cells were arranged more orderly and densely the mountaintop shrub leaves, the relative degree of stomatal opening in the mixed forest samples was higher. Transmission electron micrographs revealed that the numbers of chloroplasts and mitochondria per mesophyll cell and starch grains per chloroplast in the mountaintop shrubs were higher than those found in the conifer and broad-leaf mixed forest samples. These findings illustrate that A. shikokiana efficiently uses environmentally limited resources to adapt to different living environments. The study reveals that the mechanisms that underlie the response of A. shikokiana to heterogeneous habitats involve physiological and structural variations, thus providing a theoretical basis for future study.
\end{abstract}

\section{Hosted file}

FinalFile_a.s manuscript.docx.docx available at https://authorea.com/users/298601/articles/ 427911-physiological-and-structural-adaptability-of-anemone-shikokiana-leaves-toheterogeneous-habitats 